\title{
Evaluation of an Ayurvedic formulation in clinical recovery of COVID-19 patients: A placebo controlled pilot study among moderate-severe patients
}

\author{
Abhishek Arun \\ Atharva Multi speciality Hospital \& Research Centre \\ Amit Gupta \\ Centre for Diabetes care, Noida

\section{Saumya Subramanian} \\ SRI SRI INSTITUTE FOR ADVANCED RESEARCH \\ Divya Kanchibhotla ( $\sim$ divya.kanchibhotla@artofliving.org ) \\ SRI SRI INSTITUTE FOR ADVANCED RESEARCH
}

\section{Research Article}

Keywords: Ayurveda, COVID-19, early recovery, NOQ19, Moderate

Posted Date: December 27th, 2021

DOI: https://doi.org/10.21203/rs.3.rs-1169591/v1

License: (c) (i) This work is licensed under a Creative Commons Attribution 4.0 International License. Read Full License 


\section{Abstract}

Introduction: The COVID-19 pandemic is a global health crisis that has affected millions of people worldwide. Apart from measures taken to reduce the transmission of virus and provide symptomatic relief to patients, a potent drug targeted against the virus is required to manage the disease. Traditional medicines with an array of phytochemicals and potent anti-viral properties, can provide alternate solution in the therapeutic cure of COVID-19.

Objective: The present study investigates the efficacy of an Ayurvedic formulation, NOQ19, on the rate of recovery and clinical improvement among moderate-severe COVID-19 infected patients who were not on a ventilator or in intensive care unit.

Methods: A placebo controlled randomized study design, with 1:1 ratio between the intervention and placebo arms was adopted. The participants were provided their respective intervention along with the standard of care treatment. A follow up was conducted on Day 7 and Day 10 to assess the rate of recovery and clinical improvement. Rate of recovery was the primary outcome measured and was determined by a negative result on the RT-PCR test. The secondary outcomes included clinical improvement among patients and were measured using blood biomarkers.

Results: The NOQ19 arm had a higher percentage of population who turned RT-PCR negative on Day 7 (23\%) when compared to the placebo arm (11\%). Further subgroup analysis demonstrated that for both moderate and severe patients, there were a greater percentage of population who were RT-PCR negative in the NOQ19 arm compared to the placebo arm at Day 7: (NOQ-19: $22.22 \%$, Placebo: $14.29 \%$ for moderate patients), (NOQ19: 23.08\% Placebo: 0\% for severe patients). By Day 10, both populations depicted an almost similar percentage of the population who were RT- PCR negative. Both the groups demonstrated an improvement in blood biomarkers, although the improvement was greater in the NOQ19 arm. None of the patients in the intervention arm reported any adverse events.

Conclusion: The results of this pilot RCT indicates that NOQ19 along with standard of care treatment can be considered as an effective therapy for COVID-19 and aid early recovery from the disease.

\subsection{Introduction}

Coronavirus disease (COVID-19) is an infectious disease caused by the SARS-CoV-2 virus. The rapidly growing outbreak of COVID-19 and resulting public health crisis has garnered worldwide attention 1 , affecting 200 nations across the world2. COVID-19 was declared a pandemic by the World Health Organization (WHO) on March 11, 20203. Supportive therapy such as ventilation to optimize oxygen saturation and fluid management to intercept respiratory failure are the mainstay of current COVID-19 management4. Currently, there is a need for an antiviral drug with a targeted approach towards the virus that can reduce the viral load or cease viral replication5.

Ayurveda, an Indian traditional system of medicine, recommends several potent herbs and associated formulations for the prophylactic and therapeutic management of COVID-196. Our study focuses on an Ayurvedic polyherbal formulation containing well-known popular Ayurvedic drugs such as Ashwagandha 
(Withania somnifera), Yashtimadhu (Glycyrrhiza glabra), Pippali (Piper longum), and Guduci (Tinospora cordifolia). Several of these drugs have been recognized for their antiviral potency7-12. Phytochemical analysis of multiple Ayurvedic herbs used for their antimicrobial properties, demonstrates the presence of phenols, alkaloids and flavonoids, which contribute towards their antimicrobial activity13. A review of literature on individual components and their phytoconstituents highlights the importance of blending selected herbs into a formulation14. In silico studies on Ashwagandha (Withania somnifera) have demonstrated its antiviral properties against SARS-CoV-215-16. Among its chief phytochemical components are Withnolides, which play an active role in inhibiting viral entry into the host cells. Potential targets of Withnolides include TMPRSS2 and Mpro receptor protein and viral proteins. These proteins play an important role in viral entry and viral replication inside the host cell17. Another molecular docking study of Ashwagandha (Withania somnifera), Guduchi (Tinospora cordifolia) and Shatavari (Asparagus racemosus) demonstrated that the combined effect of phytochemicals from the three herbs target against the spike protein, main protease and RNA dependent RNA polymerase enzyme of the virus required for replication18. Yashtimadhu (Glycyrrhiza glabra), another potent antiviral drug, has been studied in silico for its mechanism of action. In silico studies highlight glycyrrhizin as one of the best antiviral chemicals against SARS-CoV-219. An animal study also highlights that glycyrrhizin plays an important role in reducing the number of Angiotensin converting enzyme 2 (ACE2) receptors in the lung tissue, which is the main binding site of SARS- CoV-2 in humans20.

Other components of NOQ19 such as Giloy (Tinospora cordifolia), Bhumiamla (Phyllanthus fraternus), Vasaka (Adhatoda vasica), Bhunimba (Andrographis paniculata) and Haridra (Curcuma longa) contain phytochemicals that target the Mpro protein of the virus. Amritoside and Apigenin-6- C-glucosyl7-0-glucoside from Giloy (Tinospora cordifolia), and Pectolinarin and Astragalin from Bhumiamla (Phyllanthus fraternus) are among the few compounds that have demonstrated the highest docking ability with Mpro protein21. Vasaka (Adhatoda vasica) is a well-known anti- inflammatory, antiviral and antitussive herbal drug.22 Known for its antithrombotic properties, Bhunimba (Andrographis paniculata) prevents blood clotting. Blood clotting is one of the severe clinical presentation of SARS-CoV-2 virus23 and antithrombotic properties of Bhunimba (Andrographis paniculata) can reduce blood clotting. A common spice used in Indian cooking with excellent antiviral properties is Tumeric or Haridra (Curcuma longa)24. Curcumin, the chief component of Haridra is a phenolic compound which has an inhibitory effect against Toll-like receptors, inflammatory cytokines and chemokines.25-26. This is crucial in COVID-19 since it can regulate the cytokine levels and inhibit the cytokine storm.

A previous in vitro analysis of NOQ19 formulation demonstrated a $100 \%$ antiviral efficacy at $0.9 \mathrm{mg} / \mathrm{ml}$ against SARS-CoV-2 virus in a Vero E6 cell based assay27. Further evaluation of NOQ19 in an in vivo model using Syrian golden hamsters demonstrated a 78.2\% efficacy and no toxicity in animals. The gross lung pathology and body weight reduction improved significantly in the NOQ19 group of hamsters28. The present study aims to determine the clinical efficacy of the above mentioned Ayurvedic herbs in a formulation, namely NOQ19, in recovery from COVID-19. Based on the previous literature, in vitro and in vivo studies on NOQ19, we hypothesize a strong therapeutic activity of this polyherbal formulation in COVID-19 patients.

\subsection{Methods}




\subsection{Trial design}

The present study was a pilot, single centre, double blind, randomized control trial. The study was approved by the Institutional ethics committee of Atharva multi-speciality hospital and research, with the registration number IEC/04/17/21/01. The study protocol was in compliance with the Helsinki Declaration and Good Clinical Practice. Adults presenting with moderate to severe COVID-19 infection, without requiring ventilation, at the OPD or IPD were screened for eligibility and invited to participate in the study. Informed consent was obtained from patients willing to

participate in the trial. The study was conducted from 19th April to 28th May, 2021. Each patient was monitored for 14 days from the time of enrollment. The study has been registered at Clinical Trial Registry India after the completion of this pilot study with registration number CTRI/2021/10/037423

\subsection{Participants}

A total of 40 patients who presented to the OPD/IPD in Atharva Multi-speciality hospitals were recruited for the study. A total of 22 patients were enrolled in the NOQ19 arm while 18 in the Placebo arm. All enrolled patients presented with symptoms of COVID-19 and tested positive on RT-PCR. The eligible participants (based on the eligibility criteria listed below) were informed about the research study. Medical history of the patients was documented and baseline parameters were assessed.

\subsubsection{Inclusion Criteria}
a) Age 21 to 75 years
b) Symptomatic COVID-19 infected patients with or without comorbidities
c) Reported to OPD within three days of onset of symptoms
d) Willing to take AYUSH treatment

\subsubsection{Exclusion Criteria}

a) Not willing to give consent/ participate in the clinical trial

b) Age less than 20 years or more than 75 years

c) Patients with chronic comorbidities like diabetes, chronic heart conditions and HIV/AIDS

d) Pregnant and lactating mothers

e) Patients on immunosuppressive therapy

f) SARS-CoV-2 infected patients on ventilation or in ICU

\subsection{Intervention:}

Patients enrolled in the study were provided respective intervention along with the standard of care treatment. The patients received a bottle containing 90 tablets each, as per their intervention. They were advised to take two tablets (500mg each) TID (thrice a day), under supervision (if IPD), along with the standard of care treatment. Compliance was monitored by the study team daily for each patient individually, in person for the patients admitted in the hospital while out patients were monitored telephonically.

\subsection{1 : NOQ19 preparation}


NOQ19 is a polyherbal formulation containing 19 ingredients made with 13 herbs. They are Ashwagandha (Withania somnifera) powder and extract, Bilwa (Aegle marmelos), Yashtimadhu (Glycyrrhiza glabra) powder and extract, Rasna (Pluchea lanceolata), Vasaka (Adhatoda vasica) powder and extract, Pippali (Piper longum), Haridra (Curcuma longa), Patha (Cissampelos pareira), Bhumiamla (Phyllanthus fraternus) powder and extract, Bhunimba (Andrographis paniculata) powder and extract, Saptaparna (Alstonia scholaris), Tulasi (Ocimum sanctum) and Guduci (Tinospora cordifolia) powder and extract.

NOQ 19 was procured from Sriveda Sattva Pvt Ltd, Bangalore (Sri Sri Tattva). NOQ19 was licensed by the Ministry of AYUSH, Govt. of India : license number- AUS782. All the herbs \& herbal extracts which constituted NOQ19 were subjected to quality control. All the ingredients were blended with excipients followed by granulation, drying and compression. After passing the QC test, the tablets were packed in bottles following standard procedure.

\subsubsection{Comparator group}

The placebo control group was provided with the placebo tablets (made of only starch), which were packed in bottles identical to the NOQ19 bottle.

\subsubsection{Standard Treatment of Care}

Standard of care treatment included allopathic medicines as prescribed by the Government of India29. Moderate patients admitted in the hospital were given Inj. Doxycycline, Inj. Azithromycin, Antipyretics and Supportive care i.e Fluids, Intravenous Steroids like Dexamethasone, Supportive oxygen therapy, Nebulization, Vitamin $C$ and Zinc. Patients who were clinically stable were treated on OPD basis and did not require hospitalization. They were given Tab Doxycycline $100 \mathrm{mg}$ BD for 5 Days, Azithromycin (500mg)- one tablet, OD, for 5 days, Vitamin C one tab TID, Tab Ivermectin 12mg OD for 3 days, Zinc, one tablet, OD and Paracetamol $(500 \mathrm{mg})$ when required.

\subsection{Outcomes}

After enrollment, the patients were monitored for 14 days. All patients were followed up for RT- PCR test on Day 0, Day 7, Day 10 to monitor viral load changes. One nasal and one throat swab were obtained for RT-PCR analysis.

\subsubsection{Primary Outcome:}

The primary outcome was the rate of recovery from COVID-19 based on RT-PCR negative test results.

\subsubsection{Secondary Outcomes :}

Secondary outcomes included clinical improvement among the patients as evaluated by blood biomarkers and chest CT score. Inflammation markers (CRP), antithrombotic activity (D-Dimer), cytokine levels (IL-6), HRCT chest score and S-Ferritin were measured. The blood parameters and immune markers were measured on Day 0 and Day 10 to observe any significant changes.

\subsection{Sample size calculation}


Being a pilot study, as a rule of thumb, the sample size was restricted to 50 in number 30 . However, due to the decrease in the number of patients in the IPD/OPD ward, the study was discontinued at 40 patients.

\subsection{Randomization and blinding}

The participants were randomized into respective study groups. A computer-generated sequence of randomization was adopted for the study. Based on their inflow, the patients were allocated numbers sequentially and thereafter randomized into groups. All the subjects were randomized in a 1:1 ratio between the intervention and placebo arm. The appropriate intervention was administered by the duty doctor who was not involved in the study. The data collector, operator and lab technicians were blinded to the intervention along with the subjects.

\subsection{Statistical analysis}

The baseline characteristics of both study groups were reported as proportion/ mean (SD). Data analysis was conducted using the intention to treat (ITT) approach. Data was compiled and analyzed using Microsoft Excel 2019 (16.0.12026.20334) 32-bit. Data was tested for normality using IBM SPSS statistics software V 2.0. The blood parameters were evaluated by descriptive statistics. Association between variables of qualitative nature was tested by Chi-square test. Fisher's Exact test was used when the value of $20 \%$ of cells of expected frequencies were $<5$ at the level of

0.05 significance. Association between variables of quantitative nature was tested by Student's T Test (paired and unpaired) wherever appropriate.

\subsubsection{Subgroup analysis}

Based on the Government of India guidelines, the patients were categorized as moderate or moderate-severe without ventilation based on $\mathrm{SpO} 2$ rate. This was further confirmed with Chest CT score, since all the patients also underwent a chest CT to assess the severity of illness31.

\subsection{Results}

The study compared the COVID-19 recovery, as measured by RT-PCR and blood biomarkers, between the intervention NOQ19 arm and placebo arm. Attrition rate in both the study groups was zero.

Table 1 demonstrates the demographic characteristics of the patients enrolled in the study. No significant differences in age or gender distribution were observed between the study groups. The two groups were also similar in height, weight, blood pressure and pulse rate at the time of enrollment. Vaccination status of the patients was also captured to analyse any change in response of the intervention between the partially and fully vaccinated groups. A significant difference was noted in the HR-CT score, SpO2 values, temperature and respiratory rate between the two groups at the time of enrollment..

According to Berkson's bias, due to the increased rate of exposure during the study enrollment and likelihood of patients being admitted in the hospital, the intervention arm had more patients with severe or advanced symptoms than the placebo arm from the start of the study.32 This resulted in a higher number of patients being severe in the NOQ19 arm than in the placebo arm. Although patients were classified as severe, none of 
them were in ICU or on ventilation. At baseline, the NOQ19 arm had $41 \%$ moderate patients and $59 \%$ patients classified with severe disease, none of whom were on ventilation, while the placebo group consisted of $78 \%$ moderate and $22 \%$ patients classified with severe disease.

Table 1

Demographic characteristic of the patients in the study arms

\begin{tabular}{|c|c|c|c|c|}
\hline Demography & & NOQ19 (n=22) & Placebo $(n=18)$ & p-value \\
\hline \multirow[t]{3}{*}{ Severity of disease $n(\%)$} & Mild & 0 & 0 & \\
\hline & Moderate & $9(41 \%)$ & $14(78 \%)$ & \\
\hline & Severe & $13(60 \%)$ & $4(22 \%)$ & \\
\hline \multirow[t]{3}{*}{ Age } & Mean (SD) & $53.64(16.23)$ & $44.44(16.13)$ & \multirow[t]{3}{*}{$0.08^{*}$} \\
\hline & Median & 55.5 & 42 & \\
\hline & Range & $22-78(56)$ & $21-75(54)$ & \\
\hline \multirow[t]{2}{*}{ Gender $\mathrm{n}(\%)$} & Male & $9(59 \%)$ & $9(50 \%)$ & \multirow[t]{2}{*}{0.57} \\
\hline & Female & $13(41 \%)$ & $9(50 \%)$ & \\
\hline Height Mean (SD) & & 167.2(6.8) & $166.9(7.3)$ & 0.92 \\
\hline Weight Mean (SD) & & $70.2(14.2)$ & $73.6(9.8)$ & 0.39 \\
\hline Pulse Rate (per min.) & & $99(14.1)$ & $91(13.8)$ & 0.10 \\
\hline \multicolumn{5}{|l|}{ Mean (SD) } \\
\hline \multicolumn{4}{|l|}{ (per min.) Mean (SD) } & 0.14 \\
\hline Temperature Mean (SD) & & $99.6(1.3)$ & $98.5(0.9)$ & $0.003^{*}$ \\
\hline $\mathrm{SpO2}$ & & $90.09(4.71)$ & $93.89(4.1)$ & $0.02 *$ \\
\hline Mean (SD) & & & & \\
\hline
\end{tabular}




\begin{tabular}{|c|c|c|c|c|}
\hline \multirow[t]{2}{*}{ Blood Pressure Mean (SD) } & Systolic & $134(16.1)$ & $128(20.2)$ & 0.29 \\
\hline & Diastolic & $80(10.8)$ & $77(10.7)$ & 0.39 \\
\hline \multirow[t]{2}{*}{ CT- Score n(\%) } & Moderate & $9(41 \%)$ & $14(78 \%)$ & \multirow[t]{2}{*}{$0.03^{*}$} \\
\hline & Severe & $13(60 \%)$ & $4(22 \%)$ & \\
\hline \multirow[t]{3}{*}{ Vaccination (n) } & Full Vaccination & 2 & 3 & \multirow[t]{3}{*}{$<0.001^{* *}$} \\
\hline & Non- Vaccination & 14 & 13 & \\
\hline & Partial Vaccination & 6 & 2 & \\
\hline Smoker (n) & & 5 & 4 & 0.969 \\
\hline Alcohol (n) & & 4 & 3 & 0.9 \\
\hline
\end{tabular}

* $p$ value $<0.05$ - Significant

** $p$ value $<0.001$ - highly significant

Table 2 demonstrates the inter and intra group analysis of COVID-19 recovery and viral load reduction as measured by RT-PCR negativity rate at different time points. Among the NOQ19 arm, 23\% $(n=5)$ of the patients were negative on Day 7 while only $11 \%(n=2)$ patients were negative in the placebo group. However on Day 10 , the number of patients who were negative in the intervention arm was $37 \%(n=8)$, and $50 \%(n=9)$ in the placebo arm. At both the time points the difference was not statistically significant between the groups. However, when the patients were grouped by severity of disease (moderate and severe), and analysed thereafter, a significant increase in the number of patients turning RT-PCR negative on day 7 was observed among both moderate and severe patients in the NOQ19 arm. Among the severe patients, the NOQ19 arm demonstrated an early recovery.

Table 2

Percentage Population with RT-PCR Negative test at Day7 and Day 10

\begin{tabular}{|lllll|}
\hline RT-PCR Negative Test & $\begin{array}{l}\text { NOQ19 }(\mathbf{n = 2 2}) \\
\mathbf{n}(\%)\end{array}$ & $\begin{array}{l}\text { Placebo Arm }(\mathbf{n = 1 8}) \\
\mathbf{n}(\%)\end{array}$ & Intergroup p value \\
\hline \multirow{3}{*}{ Total Population } & Day 0 & $0(0 \%)$ & $0(0 \%)$ & \\
\cline { 2 - 5 } & Day 7 & $5(23 \%)$ & $2(11 \%)$ & 0.423 \\
\cline { 2 - 5 } & $p$ value (Day 0 vs. 7) & $0.048^{*}$ & 0.48 & \\
\hline Day 10 & $9(41 \%)$ & $9(50 \%)$ & 0.523 \\
\hline$p$ value (Day 0 vs.10) & $0.004^{*}$ & $0.0001^{* *}$ & \\
\hline
\end{tabular}




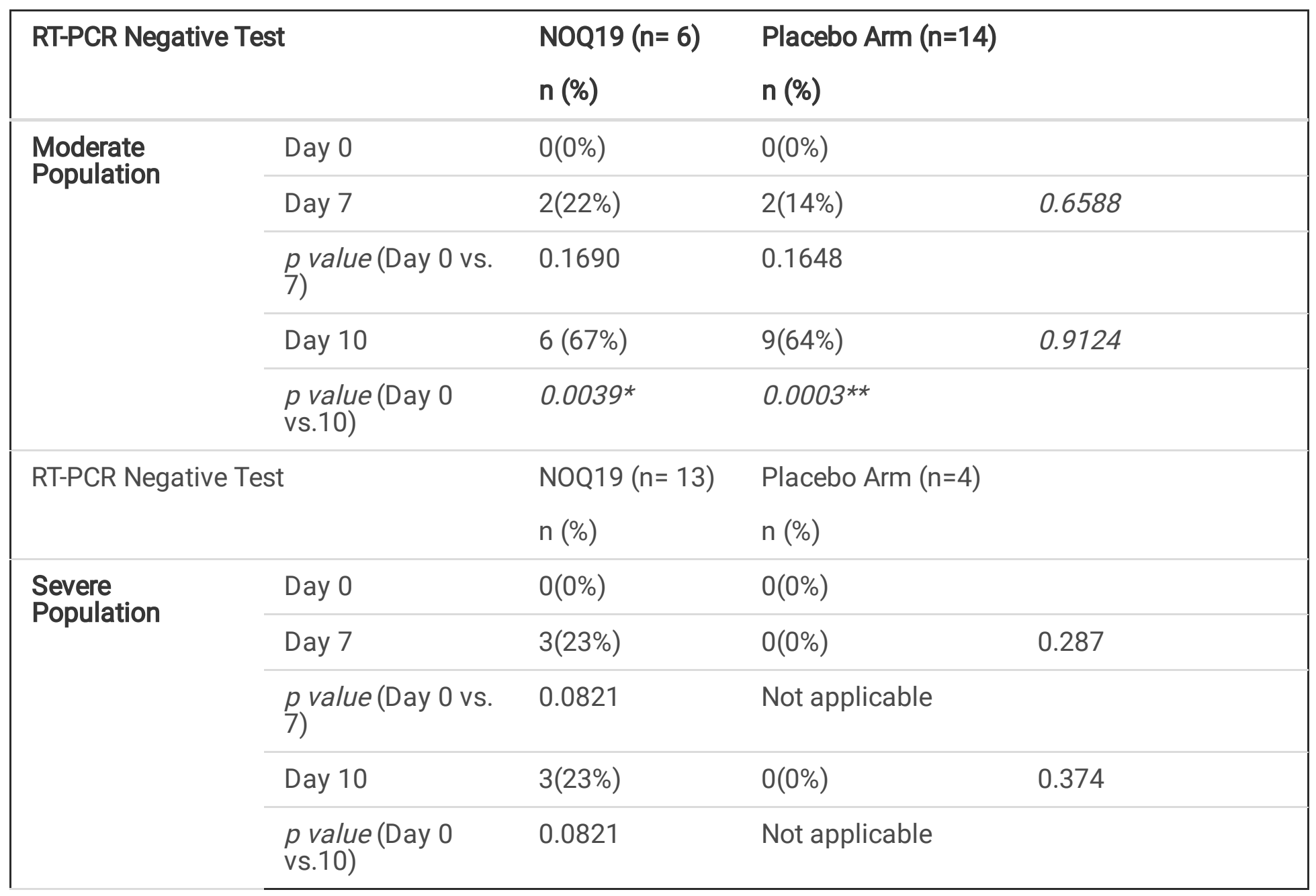

* $p$ value $<0.05$ - Significant

** $p$ value $<0.001$ - highly significant

Table 3 depicts the blood biomarker progression in both the study arms. Significant differences in temperature, immune markers and blood parameters were noted between the two groups on the day of admission. The values for IL-6, a cytokine marker, were significantly higher in the NOQ19 group as compared to placebo group at baseline $(p=0.02)$. IL- 6 showed a significant reduction from day 0 to day 10 in both the arms (intra group $p<0.001)$. However, the mean reduction was greater in the NOQ19 arm (2.2 units) as compared to placebo (1 unit). Similar trends were observed for S. Ferritin, CRP and D-Dimer. The baseline values for all the markers were much higher in the NOQ19 arm than the placebo arm. The intragroup $p$ values were significant for all markers in both arms, except D-Dimer, signifying that both the arms showed an improvement. However for all clinical markers, NOQ19 showed a greater improvement as seen by the mean difference.

Due to the differences in severity of disease between the two arms, the data was further divided into moderate and severe groups. It was analysed individually for both the arms because the sample size in each sub-group varied between the arms. Table 4 demonstrates the difference in blood parameters in severe patients among the NOQ19 and placebo arm. Except D-dimer, all the blood markers significantly improved by Day 10 in the 
NOQ19 arm. However, a significant improvement was not noted in the placebo arm, probably because of small sample size. Therefore, the data was not presented.

Table 3

Blood Parameter Inter and Intra group Analysis at Day 0, Day7 and Day 10

\begin{tabular}{|c|c|c|c|c|}
\hline Parameters & & NOQ19 (n=22) & Placebo Arm $(n=18)$ & Intergroup p-value \\
\hline \multirow[t]{5}{*}{ IL-6 } & Day 0 Mean (SD) & $10.3(6.7)$ & $6.4(3.0)$ & $0.02^{*}$ \\
\hline & DAY10 & $8.1(5.5)$ & $5.4(2.8)$ & $0.005^{*}$ \\
\hline & Mean (SD) & & & \\
\hline & IntraGroup $p$ value & $<0.001^{\star *}$ & $<0.001^{\star *}$ & \\
\hline & Mean Difference & 2.2 & 1 & \\
\hline \multirow[t]{5}{*}{ S. Ferritin } & Day 0 Mean (SD) & 892.9 ( 471.6$)$ & $649.1(386.5)$ & 0.08 \\
\hline & DAY10 & $752.5(347.2)$ & $563.1(297.5)$ & 0.071 \\
\hline & Mean (SD) & & & \\
\hline & IntraGroup $p$ value & $0.014^{*}$ & $0.001^{* *}$ & \\
\hline & Mean Difference & 140.4 & 86 & \\
\hline \multirow[t]{5}{*}{ D-dimer } & Day 0 Mean (SD) & $748.1(394.6)$ & $497.3(261.9)$ & $0.021 *$ \\
\hline & DAY10 & $641.1(283.3)$ & $445.8(225.3)$ & $0.02^{*}$ \\
\hline & Mean (SD) & & & \\
\hline & IntraGroup $p$ value & 0.113 & $0.045^{\star}$ & \\
\hline & Mean Difference & 107 & 51.5 & \\
\hline \multirow[t]{5}{*}{ CRP } & Day 0 Mean (SD) & $6.7(3.7)$ & $4.8(2.8)$ & 0.073 \\
\hline & DAY10 & $5.0(3.0)$ & $3.4(1.6)$ & $0.04^{*}$ \\
\hline & Mean (SD) & & & \\
\hline & IntraGroup $p$ value & $0.001^{* *}$ & $0.015^{\star}$ & \\
\hline & Mean Difference & 1.7 & 1.4 & \\
\hline
\end{tabular}

* $p$ value $<0.05$ - Significant

** $p$ value $<0.001$ - Highly Significant

TABLE 4: Analysis of Blood parameters among Severe patients in both the arms $n=16$ 


\begin{tabular}{|llll|}
\hline & Day 0 Mean (SD) & Day 10 Mean (SD) & p value (intragroup) \\
\hline D-Dimer & $956.16(358.42)$ & $777.07(249.54)$ & 0.116 \\
\hline CRP & $8.45(3.5)$ & $5.80(3.0)$ & $0.001^{* *}$ \\
\hline IL-6 & $12.86(7.3)$ & $9.54(6.3)$ & $0.0003^{* *}$ \\
\hline Ferritin & $1118.15(446.99)$ & $897.23(314.22)$ & $0.0016^{*}$ \\
\hline
\end{tabular}

* $p$ value $<0.05$ - Significant

** $p$ value $<0.001$ - Highly Significant

The intervention group reported no adverse events or side effects upon taking NOQ19 daily.

\subsection{Discussion}

Natural herbs have been widely utilized for viral flu and other seasonal infections. However, there is a lack of research studies on the efficacy of herbs in the management of COVID-19 patients33. To the best of our knowledge, this is the first pilot, double blind randomized clinical trial evaluating the 19 ingredient polyherbal ayurvedic formulation, NOQ19, for the therapeutic management of moderate-severe COVID-19 patients. The results demonstrated a significantly early recovery among the NOQ19 arm. All the blood markers demonstrated a marked level of improvement in both the study arms. However, it was noted that the improvement was consistently higher in the NOQ19 arm. Although the patients were randomized to different groups via computer generated sequence of randomization, baseline evaluation of clinical markers in both the arms demonstrated that the intervention arm had a higher percentage of severely infected patients than the placebo arm. Our study noticed an improvement in the rate of recovery even among the severe patients in the NOQ19 arm. However, the placebo arm did not show improvement in any severe patients until day 10. Ayurvedic medicines are usually tested in mild cases for their efficacy. Our study is the first of its kind to examine the effect of NOQ19 in moderate-severe patients who were not on ventilation or ICU.

In addition to objective marker analysis, we were interested in evaluating any side effects or adverse events that may require discontinuation of the drug in humans. There were no dropouts, side effects or adverse events in the NOQ19 arm.

In an earlier open label feasibility study on the NOQ19 polyherbal formulation, similar results were noticed with respect to RT-PCR conversion to negative. Out of 161 patients enrolled in the study, $74 \%$ of the patients turned RT-PCR negative by Day 534. In vitro and in vivo studies $27-28$ on the effectiveness of the polyherbal formulation, NOQ19 also showed significant viral load reduction. In the in vitro analysis of NOQ19, a 100\% viral load reduction was noted in the Vero E6 SARS-CoV-2 infected cell line. The in vivo animal study on infected Syrian golden hamsters demonstrated a $78.2 \%$ viral load reduction in hamster lungs compared to the infected untreated group. Among the researched herbs, Yashtimadhu (Glycyrrhiza glabra) has been extensively studied via in silico studies for presence of phytochemicals possessing antiviral properties. Glycyrrhizin, a 
component of Yashtimadhu (Glycyrrhiza glabra) has been identified as the chief component responsible for inhibiting the attachment of SARS-CoV-2 spike protein with the ACE-2 receptors35. Clinical trials on Ashwagandha (Withania somnifera) have demonstrated its immunogenic, anti-inflammatory and adaptogenic properties36. Another placebo controlled trial on Ashwagandha (Withania somnifera), Giloy (Tinospora cordifolia) and Tulasi (Ocimum sanctum) demonstrated that $71.1 \%$ of patient population recovered from COVID-19 on day 3 in the treatment group. However, only $50 \%$ of patients recovered from COVID-19 in the placebo group. Also, the study noticed that serum CRP level, IL-6 and TNF alpha were 20 times lesser in the intervention group than the placebo group on day 737. Another study quoted that patients receiving an Ayurvedic regimen of Dasamoolkaduthrayam Kashaya and Guluchyadi Kwatham presented with early recovery and reduced length of stay in the hospital38. Upon further inspection, it was noted that the above mentioned drugs had Bilwa (Aegle marmelos), Pippali (Piper longum), Vaska (Adhatoda vasica) and Guduchi (Tinospora cordifolia), in common with NOQ19 formulation. Although our study did not capture the details regarding the duration of hospital stay, the objective markers represent that patients in the NOQ19 arm recovered well and therefore, did not require a long hospitalization. Another AYUSH drug, Kabasura Kudineer, has been well studied for its efficacy in symptom recovery and therapeutic cure against COVID-19. Kabasura Kudineer and Nilavembu Kudineer along with the standard of care treatment were compared against standard treatment alone for COVID-19. The authors noticed that patients in the intervention group took only 2.7 days approximately to turn asymptomatic while those with standard of care treatment took 4.2 days 39 . Our study demonstrated that the Ayurvedic formulation, NOQ19, expedited COVID-19 recovery, viral load reduction and clearance, as measured by RT-PCR negative test. Correlating with the viral clearance, the immune markers and inflammatory markers also demonstrated a significant improvement among patients with severe disease. One of the key limitations of the study is the small sample size. Further randomized control studies on the formulation with larger sample sizes can evaluate the effectiveness of the drug in COVID-19 patients.

\subsection{Conclusion}

This study evaluated the therapeutic efficacy of a 19 ingredient Ayurvedic, polyherbal formulation, NOQ19 for clinical improvement in COVID-19 patients. A higher rate of recovery was noted in the NOQ19 arm on Day 7 compared to placebo, among moderate-severe patients. The immune markers, inflammatory markers and other blood markers demonstrated a significant clinical improvement among the patients who took NOQ19, even among those with severe disease. Additionally, the patients who took NOQ19 experienced no adverse effects. This study highlights the efficacy of NOQ19, an Ayurvedic formulation, in the management of COVID 19 , when administered along with standard care of treatment. Based on this pilot study, further clinical trials can throw light on the efficacy of NOQ19 in COVID-19.

\section{Declarations}

\section{Acknowledgement}

We would like to thank Dr Ravi Reddy (Chief Science Officer) and Dr Hari Venkatesh (Head, Research \& Development - Healthcare) from Sriveda Sattva for providing NOQ19 and placebo tablets to the study site Atharva Multi Speciality and Research Center. We would also like to acknowledge Dr. Somya Ramrakhyani for language edits.

Page 12/16 


\section{Conflict of Interest:}

All authors declare there is no conflict of interest.

\section{Funding:}

No funding was received

Data Availability: The individual patient level data can be accessed at the following link https://docs.google.com/spreadsheets/d/1keaMxG6JblvyGV1n7EMbK8Yo_WaJbAiPeDj_KTHvpiQ/edit\#gid=0

\section{References}

1. Jin Y, Yang H, Ji W, et Virology, Epidemiology, Pathogenesis, and Control of COVID-19. Viruses 2020;12(4):372.

2. Pal R, Yadav U. COVID-19 pandemic in india: present scenario and a steep climb ahead. Journal of primary care \& community health. 2020 Jul;11:2150132720939402

3. World Health Organization. Coronavirus disease (COVID-19). Available from : https:// who.int/emergencies/diseases/novel-coronavirus-2019 Accessesed on 10 sept 2021.

4. Gavriatopoulou M, Ntanasis-Stathopoulos I, Korompoki E, et Emerging treatment strategies for COVID-19 infection. Clin Exp Med 2021;21(2):167-179

5. Jacek Smereka, Lukasz Szarpak, Krzysztof J. Filipiak, Modern medicine in COVID-19 era. DOI: 5603/DEMJ.a2020.0012,Disaster Emerg Med J 2020;5(2):103-105.

6. Patwardhan B, Chavan-Gautam P, Gautam M, Tillu G, Chopra A, Gairola S, et al. Ayurveda rasayana in prophylaxis of COVID-19. Curr Sci 2020;118(8):1158-60.

7. Santhanu K, Senthil Therapeutic potential of Withania somnifera (Linn) Dunal (Ashwagandha) in historical perspective and pharmacological evidence. Annals of Ayurvedic Medicine 2021;10(2):135-47.

8. Anagha K, Manasi D, Priya L, Meera M. Scope of Glycyrrhiza glabra (Yashtimadhu) as an antiviral agent: a Int. J. Curr. Microbiol. App. Sci 2014;3(12):657-65.

9. Kumar A, Prasad G, Srivastav S, Gautam VK, Sharma N. Efficacy and Safety of Guduchi Ghan Vati in the Management of Asymptomatic COVID-19 Infection: An Open Label Feasibility medRxiv 2020.

10. Jikadara J, Roy SP. Review on Potential Herbal Remedies to Combat Covid-19. Journal of Ayurvedic and Herbal Medicine 2021;7(2):124-33.

11. Lal Turmeric, curcumin and our life: a review. Bull Environ Pharmacol Life Sci 2012;1(7):11-7.

12. Joshi RK, Setzer WN, Da Silva Phytoconstituents, traditional medicinal uses and bioactivities of Tulsi (Ocimum sanctum Linn.): a review. Am J Essent Oil Nat Prod 2017;5(1):18-21.

13. Ben-Shabat S, Yarmolinsky L, Porat D, Dahan A. Antiviral effect of phytochemicals from medicinal plants: Applications and drug delivery Drug Deliv Transl Res 2020;10(2):354-67.

14. Bone K. A Clinical Guide to Blending Liquid Herbs E-Book: Herbal Formulations for the Individual Elsevier Health Sciences 2003. 
15. Shree P, Mishra P, Selvaraj C, Singh SK, Chaube R, Garg N, Tripathi YB. Targeting COVID-19 (SARS-CoV-2) main protease through active phytochemicals of ayurvedic medicinal plants-Withania somnifera (Ashwagandha), Tinospora cordifolia (Giloy) and Ocimum sanctum (Tulsi)-a molecular docking study. Journal of Biomolecular Structure and Dynamics 2020:1-4.

16. Tripathi MK, Singh P, Sharma S, Singh TP, Ethayathulla AS, Kaur P. Identification of bioactive molecule from Withania somnifera (Ashwagandha) as SARS-CoV-2 main protease Journal of Biomolecular Structure and Dynamics 2020:1-4.

17. Borse S, Joshi M, Saggam A, Bhat V, Walia S, Marathe A, et al. Ayurveda botanicals in COVID-19 management: An in silico multi-target Plos one 2021;16(6):e0248479

18. Niraj S, Varsha A review on scope of immuno-modulatory drugs in Ayurveda for prevention and treatment of Covid-19. Plant Science Today 2020;7(3):417-23.

19. Damle Glycyrrhiza glabra (Liquorice)-a potent medicinal herb. Int J Herb Med 2014;2(2):132-6.

20. Jezova D, Karailiev P, Karailievova L, Puhova A, Murck Food Enrichment with Glycyrrhiza glabra Extract Suppresses ACE2 mRNA and Protein Expression in Rats- Possible Implications for COVID-19. Nutrients $2021 ; 13,2321$.

21. Murugesan S, Kottekad S, Crasta I, Sreevathsan S, Usharani D, Perumal MK, et Targeting COVID-19 (SARSCoV-2) main protease through active phytocompounds of ayurvedic medicinal plants-Emblica officinalis (Amla), Phyllanthus niruri Linn.(Bhumi Amla) and Tinospora cordifolia (Giloy)-A molecular docking and simulation study. Comput. Biol. Med 2021;136:104683.

22. Khanal H, Khanal U, Koirala J. Medicinal plant vasaka could be a therapeutic option for the management of COVID-19 symptoms. J Med Plants 2020;8(5):44-8.

23. Mishra SK, Sangwan NS, Sangwan RS. Phcog rev.: Plant review Andrographis paniculata (Kalmegh): A Pharmacogn Rev 2007;1(2):283-98.

24. Mathew D, Hsu Antiviral potential of curcumin. Journal of functional foods 2018;40:692-9.

25. Zahedipour F, Hosseini SA, Sathyapalan T, Majeed M, Jamialahmadi T, Al-Rasadi K, et Potential effects of curcumin in the treatment of COVID-19 infection. Phytotherapy Research 2020;34(11):2911-20.

26. Soni VK, Mehta A, Ratre YK, Tiwari AK, Amit A, Singh RP, et al. Curcumin, a traditional spice component, can hold the promise against COVID-19?. European Journal of 2020:173551.

27. Kanchibhotla D, Subramanian S, Reddy R, KR HV, Pathania M. An In-vitro evaluation of a polyherbal formulation, against SARS-CoV-2.

28. Kanchibhotla D, Subramanian S, KR HV. To study the in-vivo efficacy and safety of AYUSH polyherbal formulation among COVID-19 infected Syrian gold

29. Revised Guidelines on Clinical Management of COVID-19. https://www.mohfw.gov.in/ pdf/RevisedNationalClinicalManagementGuidelineforCOVID1931032020.pdf. Acessed on 30/09/2021

30. Whitehead AL, Julious SA, Cooper CL, Campbell MJ. Estimating the sample size for a pilot randomised trial to minimise the overall trial sample size for the external pilot and main trial for a continuous outcome Statistical methods in medical research 2016;25(3):1057-73. 
31. NATIONAL CLINICAL MANAGEMENT PROTOCOL COVID-19. https:// w w w . m o h f w . g o v . i n / p d f / pdf. Accessed on 30/09/2021

32. Berkson's bias. Oxford Reference. Available from https://oxfordreference.com/view/ 10.1093/oi/authority.20110803095500748. Accessed on 13 Sept. 2021

33. Prakash P, Meena R, Stanley AB, Swetha SU, Govindaraju K, Durgasruthi PU, et EVIDENCE-BASED traditional Siddha formulations for prophylaxis and management of respiratory symptoms in COVID-19 pandemic-a review. Biocatalysis and Agricultural Biotechnology 2021:102056.

34. Kanchibhotla D, Harsora P, Subramanian S, Venkatesh Efficacy of a polyherbal formulation in the treatment of SARS CoV-2 disease: An open labelled feasibility study.

35. Hoever G, Baltina L, Michaelis M, Kondratenko R, Baltina L, Tolstikov GA, et al. Antiviral activity of glycyrrhizic acid derivatives against SARS- J Med Chem 2005;48(4):1256-9.

36. Gupta GL, Rana Withania somnifera (Ashwagandha): a review. Pharmacognosy Reviews. 2007;1(1).

37. Shree P, Mishra P, Selvaraj C, Singh SK, Chaube R, Garg N, et al. Targeting COVID-19 (SARS-CoV-2) main protease through active phytochemicals of ayurvedic medicinal plants- Withania somnifera (Ashwagandha), Tinospora cordifolia (Giloy) and Ocimum sanctum (Tulsi)-a molecular docking J Biomol Struc. Dyn 2020;26:1-4.

38. Wanjarkhedkar P, Sarade G, Purandare B, Kelkar D. A prospective clinical study of an Ayurveda regimen in COVID 19 patients. Journal of Ayurveda and integrative medicine.

39. Srivastava A, Rengaraju M, Srivastava S, Narayanan V, Gupta V, Upadhayay R, et al. Efficacy of Two Siddha Poly herbal Decoctions, Nilavembu Kudineer and Kaba Sura Kudineer, along with Standard Allopathy Treatment in the Management of Mild to Moderate Symptomatic COVID-19 Patients-a DoubleBlind, Placebo Controlled, Clinical 2021

\section{Figures}




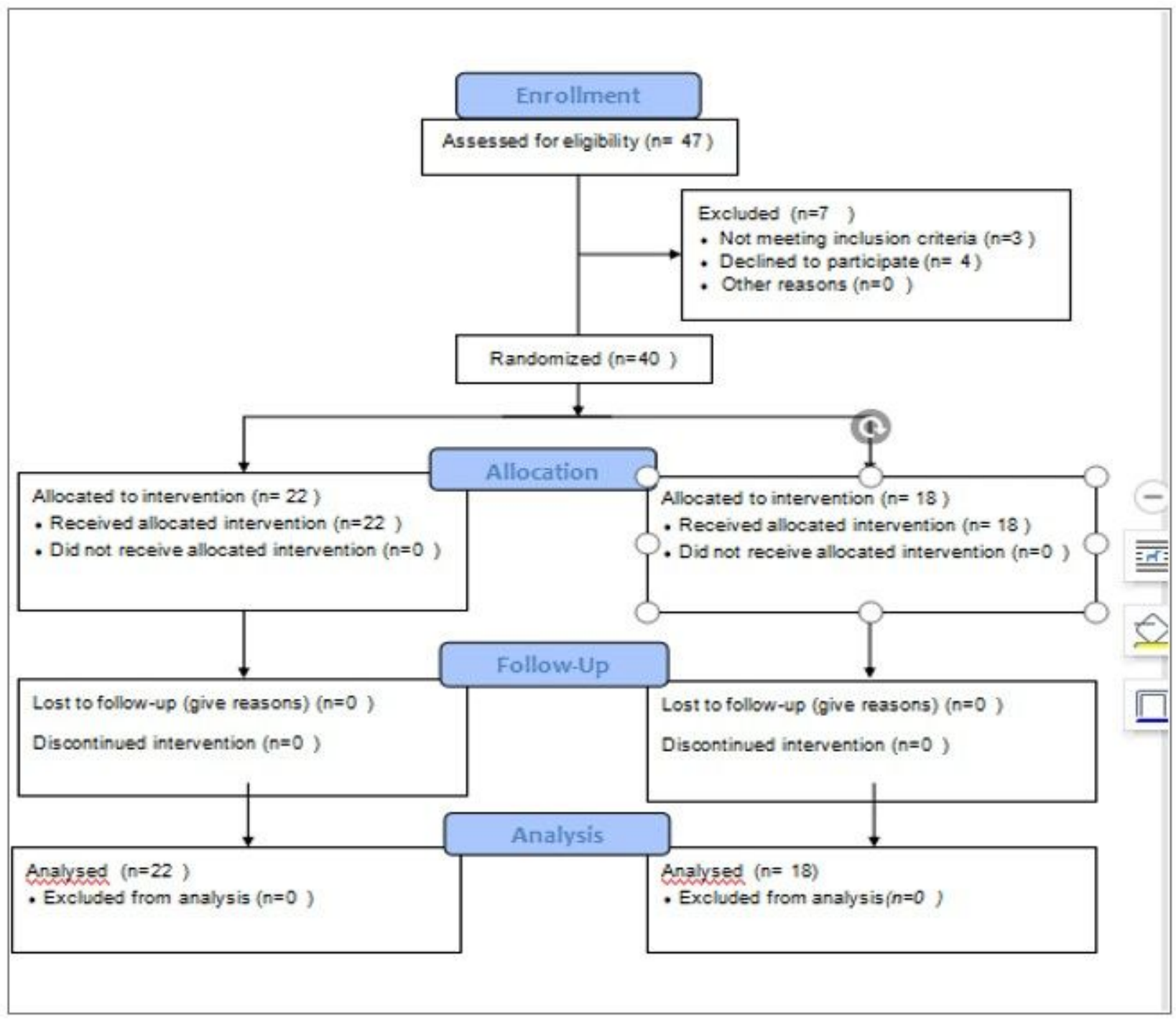

Figure 1

CONSORT Flow Diagram 